\title{
Religion et Révolution en Alsace
}

\section{Claude Muller}

\section{(2) OpenEdition \\ Journals}

Édition électronique

URL : https://journals.openedition.org/ahrf/1514

DOI : 10.4000/ahrf.1514

ISSN : 1952-403X

Éditeur :

Armand Colin, Société des études robespierristes

Édition imprimée

Date de publication : 1 septembre 2004

Pagination : 63-83

ISSN : 0003-4436

\section{Référence électronique}

Claude Muller, «Religion et Révolution en Alsace», Annales historiques de la Révolution française [En ligne], 337 | juillet-septembre 2004, mis en ligne le 15 septembre 2007, consulté le 23 avril 2022. URL : http://journals.openedition.org/ahrf/1514 ; DOI : https://doi.org/10.4000/ahrf.1514

Ce document a été généré automatiquement le 23 avril 2022.

Tous droits réservés 


\title{
Religion et Révolution en Alsace
}

\author{
Claude Muller
}

1 Extrême-Orient de la France du XVIIIe siècle, l'Alsace occupe une place à part dans le royaume. Elle connaît sur son sol les affres de la guerre ${ }^{5}$. Entrée tardivement dans le giron de la monarchie française, elle conserve des particularités d'un pays rhénan, riche d'une agriculture diversifiée ${ }^{6}$ et de son industrie naissante ${ }^{7}$. La langue allemande reste omniprésente. Religieusement, c'est un invraisemblable kaléidoscope où se côtoient catholiques, luthériens, calvinistes, juifs et anabaptistes, sans compter ceux qui refusent Dieu ${ }^{8}$. Province-frontière, l'Alsace voit, pendant la Révolution ${ }^{9}$, guerre et lutte religieuse ${ }^{10}$ s'emmêler inextricablement. On y vit dans l'atmosphère de l'émigration, de la trahison, de la fraude. Bref, tout est ici à la fois semblable et dissemblable de ce qui se passe en « vieille France ».

I - « Il faut sortir le peuple des ténèbres de l'obscurantisme » (1789-1792)

2 Le XVIIIe siècle voit incontestablement le triomphe de la croix en Alsace ${ }^{11}$. Le clergé séculier accroît ses effectifs ${ }^{12}$, les couvents ne désemplissent pas ${ }^{13}$. Symboles d'un monde plein, les églises s'avèrent trop petites pour contenir les fidèles ${ }^{14}$. À une époque où la naissance implique la compétence, les fastueux Rohan ${ }^{15}$ éblouissent l'Europe à Saverne et Strasbourg, alors que les Reinach et les Monjoie ${ }^{16}$ affirment une noblesse rhénane à Porrentruy. Pour Roland Marx ${ }^{17}$, la période prérévolutionnaire se caractérise par un éveil lent à la vie politique en l'absence d'un parlement frondeur. De fait, le Conseil souverain d'Alsace ${ }^{18}$, serviteur zélé de la monarchie, apparaît comme un instrument de la francisation et de la catholicisation ${ }^{19}$ du pays. Aube et robe se mélangent avec bonheur ${ }^{20}$. Entre une douce cohabitation et la violence évangélique, les luthériens ${ }^{21}$ développent leur identité. Juifs et calvinistes espèrent des jours meilleurs.

1) La terrible rupture des catholiques

3 Si l'historiographie alsacienne dénonce la fureur anti-religieuse et la barbarie sanguinaire, voire exhalte le culte des martyrs, les contemporains estiment que la Révolution répond à l'exigence de réformes profondes, même si l'inquiétude du clergé est palpable ${ }^{22}$. Comme l'a bien relevé Tocqueville, l'anticléricalisme en veut moins à la religion qu'aux richesses excessives de l'Église, au sol qu'elle accapare (l'abbaye cistercienne de Lucelle possède 6000 hectares), à la puissance politique ${ }^{23}$. Et Fernand 
L'Huillier remarque que, durant de longs mois, l'hostilité systématique à l'Église et à la religion reste un phénomène circonscrit ${ }^{24}$.

4 La rupture s'amorce avec la Constitution civile du clergé ${ }^{25}$, décrétée le 12 juillet 1790 , connue en Alsace en septembre, qui fait valoir une exigence radicale : transformer le clergé en un corps de fonctionnaires, à la fois dévoué à la Révolution et rapproché de la pureté évangélique, à la fois patriote et gallican, en s'appuyant sur le principe de l'élection. Cette mesure se répand en même temps que des rumeurs les plus folles, des pamphlets, des proclamations épiscopales et des contre-proclamations administratives. Louis René Édouard de Rohan Guémené ${ }^{26}$, franc-maçon ${ }^{27}$, s'y oppose, ralliant à lui la quasi-totalité de son clergé ${ }^{28}$, malgré sa position fragilisée par l'affaire du collier de la reine.

5 Parmi d'autres exemples, voici ce que déclare le curé de Saverne, Jansen, le 27 janvier 1791 : «Plutôt souffrir la pauvreté la plus humiliante, plutôt voir tous les miens réduits à n'avoir pour toute nourriture que ce qu'on leur donnera au nom de Dieu que de scandaliser le troupeau confié à mes soins que de m'exposer aux reproches des fidèles et à ceux de ma conscience que de déshonorer le caractère sacré dont je suis revêtu, que de me rendre indigne de votre estime [...] Je serai jusqu'au dernier soupir fidèle à la nation, à la loi et au roi et soumis à toute constitution politique en ce qu'elle ne sera pas opposée à la foi catholique, apostolique et romaine, dans laquelle je veux vivre et mourir ${ }^{29}$. Pour René Epp, les objectifs gallicans de la Constitution ne peuvent être partagés par le clergé ultramontain bas-rhinois.

6 L'élection de François Antoine Brendel, le 6 mars 1791 comme évêque constitutionnel $\mathrm{du}$ Bas-Rhin constitue un désastre du point de vue psychologique, car les catholiques n'admettront jamais qu'il l'a été avec des voix luthériennes ${ }^{30}$. De plus, il y a désormais deux évêques, donc un de trop. Entre le falot Brendel et le ferme Rohan, à l'abri à Ettenheimmünster, sur la rive droite du Rhin, le clergé n'hésite pas. Brendel est vite submergé par mille difficultés et, malgré l'apport d'un clergé en provenance d'Allemagne ${ }^{31}$ ouvert aux idées révolutionnaires et attiré par des places à prendre, il ne peut se soutenir, tout comme d'ailleurs le squelettique clergé constitutionnel. Et ce en dépit de l'activisme d'Euloge Schneider ${ }^{32}$.

7 En haute-Alsace, la situation apparaît différente. Certes, l'élection de l'insipide Arbogast Martin ${ }^{33}$, après le bref intermède de Jean Baptiste Gobel ${ }^{34}$, élu aussi avec des voix luthériennes, se calque sur le modèle strasbourgeois, y compris avec ses répercussions négatives. Mais l'évêque, incompétent et vite dépassé, peut s'appuyer sur une base bien plus solide, car beaucoup de prêtres acceptent de prêter le serment ${ }^{35}$. À un Bas-Rhin « autrichien » s'oppose donc un Haut-Rhin « républicain », bien cerné par Jules Joachim ${ }^{36}$. La radicalisation, qui pousse à la fermeture des couvents d'hommes au printemps 1791 et les couvents de femmes à l'automne $1792^{37}$, provoque l'exode et le retour chez soi de centaines de religieux ${ }^{38}$ et de religieuses ${ }^{39}$, dans un désarroi général. "Quisquis igitur levis animo quam professus est, vita rationem, stationem sciliset Augusti nianam inconstans deserit vel deseret, Patris Nostri Augustinis filius non fuit, non est » écrit l'ex-provincial Clément Oberlé à son ami Jérôme Morel, le 2 janvier 1791, avant de se déclarer « ad mortem usque Augustinensis » ${ }^{40}$.

Que nous apprennent les chiffres, permettant de dresser une typologie des comportements de prêtres ? C'est là que débutent les difficultés. Alors que l'on pourrait s'attendre à ce que rien ne soit plus irréfutable qu'un nombre, la statistique bafouille et embrouille. Les flagrantes contradictions des surabondants états révolutionnaires ${ }^{41} \mathrm{ne}$ 
peuvent être compensées que par l'établissement de fiches d'ecclésiastiques, forcément lacunaires. Plutôt que des chiffres précis, examinons les ordres de grandeur. Fin 1791, dans le Bas-Rhin, Louis Kammerer dénombre $7,6 \%$ de constitutionnels, Timothy Tackett 9\%(40), Dominique Varry 9,6\%, avec l'ignorance des conditions dans lesquelles les prestations ont été effectuées. Pour le Haut-Rhin, Tackett propose ${ }^{42} \%$ à l'été 1791, mais Dominique Varry aboutit, à la même période, à 31,4\% de prestation de serment pur et simple et à $37,5 \%$ de serment avec restriction. Plus on progresse vers l'Alsace méridionale, plus le serment est prêté. Au sein du clergé régulier, des disparités apparaissent : 10 bénédictins sur 95 prêtent le serment (10,5\%), 7 augustins sur 62 (11,2\%), 9 dominicains sur 55 (16,4\%), etc. Chiffres à comparer avec la diversité des pourcentages d'assermentés, relevés par Timothy Tackett pour le Grand Est: Doubs $21 \%$, Moselle $32 \%$, Haute-Saône $35 \%$, Meurthe $45 \%$, Vosges $63 \%$. Et ceux, plus fiables, relevés en Provence ${ }^{43}$.

2) Les luthériens, moteurs girondins de la Révolution ?

9 À la veille de la Révolution, les protestants représentent environ 220000 personnes regroupées en 146 communautés face aux 450000 catholiques. Ils sont surtout implantés au nord de l'Alsace ${ }^{44}$, à Strasbourg et à Colmar ${ }^{45}$, alors que la calviniste Mulhouse est encore suisse. Minorité fortunée et influente, qui s'engage dans l'industrie et le commerce, car exclue des fonctions administratives de la monarchie, la communauté protestante - où les luthériens ne sont guère charitables envers les calvinistes ${ }^{46}$ - est en butte, pendant tout le XVIIIe siècle, à l'hostilité catholique. Les cahiers de doléances du clergé catholique expriment encore un vif antagonisme envers les luthériens, ce qui incite ces derniers à rédiger un cahier de doléances spécifique non prévu ${ }^{47}$.

10 Dès le 1er octobre 1789, le magistrat luthérien de Strasbourg 48 écrit à Paris : "la religion protestante est non seulement tolérée à Strasbourg, mais établie légalement en vertu des traités de paix dont la capitulation stipule expressément qu'il ne sera dérogé en rien à l'état de cette religion dans la ville et ses dépendances, qu'une égalité parfaite quant à l'exercice du culte public et l'admission aux charges sera la loi commune des religions catholique et protestante, que cette dernière religion sera maintenue dans la possession tranquille et imperturbable des biens ecclésiastiques et fondations qui lui appartiennent, tels le chapitre de Saint-Thomas, l'université, le collège SaintGuillaume " ${ }^{49}$. Le 22 mai 1790, Koch envoie à Paris un long plaidoyer pour l'égalité ${ }^{50}$.

11 Les deux premières décisions de l'Assemblée constituante, à savoir l'abolition des privilèges qui met le pasteur protestant sur le même pied que le curé catholique et la Déclaration des droits de l'homme et du citoyen qui autorise l'accès à toutes les carrières de l'État, font adhérer une majorité de protestants à la Révolution en marche ${ }^{51}$, malgré quelques résistances : ainsi, en 1791, certains pasteurs continuent à faire la prière pour le seigneur du lieu. L'adhésion se traduit par une Société des Amis de la Constitution de Strasbourg, peuplée en majorité de luthériens, des dons patriotiques plus importants dans les localités protestantes. Presque tous les rédacteurs et imprimeurs de journaux alsaciens sont protestants ${ }^{52}$.

12 Cette ardeur, incarnée par le maire de Dietrich ${ }^{53}$ et le théologien Blessig ${ }^{54}$, est reconnue par les partisans de la Révolution: "les luthériens et les calvinistes sont fermement dévoués à la patrie " ${ }^{55}$. À partir de 1792, le courant de gauche des révolutionnaires trouve un certain nombre d'adeptes chez les luthériens. Georges Frédéric Coulmann, notaire à Brumath, joue un rôle actif comme administrateur du 
district de Haguenau. Georges Dentzel, pasteur à Landau, est élu en 1792 député de la Convention. Gotthard Grimmer, pasteur à Wissembourg, député suppléant en 1795, voit son zèle jacobin célébré par le comité révolutionnaire du district de Wissembourg. Signalons tout de même la fin d'un mythe: les catholiques accusent les protestants d'acheter des biens d'Église, alors qu'eux-mêmes s'en abstiennent. Les études récentes démontrent que tout un chacun achète ${ }^{56}$. Certes le protestant Collombel acquiert des bâtiments conventuels pour y installer une fabrique, mais des milliers de catholiques s'empressent de payer, qui un rouleau de pâtisserie, qui une bêche, qui un lit provenant des biens ecclésiastiques vendus à l'encan.

$13 \mathrm{Au}$ final, cette appréciation du sommelier catholique, Jean Spielmann, à Colmar, en 1792 : «Il y avait beaucoup de mauvais sang entre les catholiques et les luthériens, ce qui me chagrinait beaucoup, parce que de pareils dissentiments ne pouvaient que nuire à la religion en général. À entendre les luthériens, la révolution ne se faisait que pour eux. Ils allaient se fourrer les pieds dans nos pantoufles et se donner du bon temps. Selon les révolutionnaires, aucune des deux religions ne valait rien et il fallait démolir toutes les églises le plus tôt possible ${ }^{57}$. Opinion personnelle ou opinion partagée?

3) Les juifs : entre espoir et expectative

Plus encore que les luthériens, les juifs constituent une «nation » à part, victime de l'opprobre de la société majoritaire et de l'enseignement du mépris. Ils sont 20000 en Alsace sur 40000 résidant en France. La majorité d'entre eux vivent du colportage, de la friperie, de la revente. Leur nombre est important dans le commerce des bestiaux, des chevaux et des grains. Ils s'adonnent au courtage d'argent (argent, revente des maisons et des terres), activité dénoncée par l'opinion publique même s'il convient de signaler que le prêt porte sur des sommes réduites et avancées pour de courtes périodes ${ }^{58}$. La plupart des cahiers de doléances font preuve d'un antisémitisme virulent. Ainsi, le clergé réuni de Colmar et de Sélestat souligne que les "rapines» des juifs sont la principale cause de la misère du peuple. Il exige que «leur étonnante pullulation soit arrêtée dans son principe et qu'il ne puisse plus être permis de contracter mariage qu'au fils aîné de chaque famille juive ».

15 Le 25 mai 1789, les représentants de la nation juive en Alsace, réunis à Strasbourg, expriment leurs vœux dans un cahier spécifique ${ }^{59}$. À la fin du mois de juillet 1789 , les paysans s'attaquent aux abbayes et aux châteaux, mais aussi aux juifs de Durmenach, Hagenthal et Hegenheim, dans le Sundgau, dont les maisons sont pillées, comme s'en fait l'écho la chronique du Colmarien Schmutz ${ }^{60}$. Vu le contexte difficile, les juifs d'Alsace députent David Sintzheim et Joseph Brunschwig pour acheminer leur propre cahier de doléances à Paris. Le texte présenté à Versailles, en août 1789, défendu par l'abbé Grégoire ${ }^{61}$, exprime que, sur le plan économique, soient supprimées les discriminations en matière de fiscalité et d'activité professionnelle.

Symbolisant l'adhésion des juifs à la Révolution, le fils aîné de Cerf Beer ${ }^{62}$, Max Beer, se fait admettre dans la garde nationale et le 20 février 1790 à la Société des Amis de la Constitution de Strasbourg, favorable aux juifs en ce début de Révolution. Mais si les juifs «portuguais, espagnols et avignonais » ${ }^{63}$ sont émancipés le 28 janvier 1790 , les juifs d'Alsace doivent attendre le 27 septembre 1791 pour obtenir les mêmes droits ${ }^{64}$. Le retard est lié au blocage entrepris par les Alsaciens, tant catholique, que luthériens. Jean-François Reubell, futur directeur du Directoire, en prend sa part : «Vous voulez donner le titre de citoyen aux juifs? Non les juifs ne sont pas des citoyens; ils forment une nation qui a ses lois particulières. Peuvent-ils être soldats? Il ne peuvent se battre 
le samedi. Artisans ? Mais ils refusent de travailler le samedi et ils ont des fêtes trop nombreuses. Si vous voulez leur donner le titre de citoyen, toute l'Alsace se révolterait ${ }^{65}$.

II - La terreur : le temps de la violence et du désenchantement (1793-1794)

La guerre aux portes de l'Alsace, voire dans sa partie septentrionale ${ }^{66}$, la radicalisation de la lutte contre la religion ${ }^{67}$, le va-et-vient incessant à la frontière, l'arrivée en Alsace de Saint-Just et Lebas, tout concourt en quelques semaines à transformer la Révolution en un mouvement sanglant.

1) L'apogée de la lutte contre le catholicisme

Quatre faits marquants sont à relever: l'instauration d'un nouveau culte, les abdications de prêtres, l'affaire d'Hirsingue et le prix du sang. Commençons par le nouveau culte. Le département du Bas-Rhin ordonne le 9 novembre 1793 « la fermeture de tous les cultes quelconques pendant la guerre». Transformée en Temple de la Raison, la cathédrale voit se dérouler le 20 novembre - soit dix jours après Paris - un culte singulier autour d'un "monument élevé à la nature ", tandis qu'on entreprend d'enlever et de briser les statues. Le 6 décembre 1793, le nouveau culte de la Raison est installé à Colmar. Sans prétendre à l'exhaustivité, signalons ce culte à Barr, Haguenau, Niederschaeffolsheim, Obernai, Rosheim, Wissembourg dans le Bas-Rhin; Belfort, Bergheim, Cernay, Dannemarie, Huningue, Husseren, Soultz, Turckheim dans le HautRhin. On se contente souvent d'une inscription «temple de la Raison » sur la porte de l'église paroissiale fermée. À Wissembourg ${ }^{68}$, se déroule "eine Eselsprocession », où l'on fait défiler des ânes portant des ornements sacerdotaux et des livres qui seront brûlés sur un bûcher. Les cérémonies du culte sont bien connues, grâce aux exemples étudiés de Belfort ${ }^{69}$ et de Haguenau ${ }^{70}$. Le 30 mai 1794, le culte de l'Être Suprême succède au culte de la Raison pour deux petits mois, sans cérémonie particulière à Colmar, où l'évêque Martin décède le 12 juin, tout un symbole. Son convoi mortuaire traverse la ville sans attirer l'attention; seuls deux pasteurs luthériens l'accompagnent...

19 En même temps qu'est instauré le culte de la "déraison ", sobriquet donné par les opposants, débute l'abdication des prêtres : Euloge Schneider, devenu accusateur public du Tribunal Révolutionnaire à Strasbourg le 20 novembre 1793 ; d'Aigrefeuille et Albert le 6 décembre 1793 à Colmar. Louis Kammerer dénombre 102 abdicataires pour le BasRhin et 68 pour le Haut-Rhin ${ }^{71}$. Nous aboutissons à un chiffre plus élevé : 114 pour le Bas-Rhin et 115 pour le Haut-Rhin ${ }^{72}$. Parmi ces prêtres abdicataires, les nombreux Allemands, ainsi Knoblauch qui renonce à « toutes les extravagances qu'il a été forcé de faire malgré lui », à ces " momeries religieuses » et «à ce délire diabolique ». Pour une abdication sincère, combien de forcées? 78 mariages de prêtres sont connus: le premier le 30 mai 1792, une vingtaine en décembre 1793, puis quatre à cinq par an. Les témoins sont souvent des ex-prêtres. En se limitant au clergé en fonction en 1793, cela fait un taux de $20 \%$. En déduisant les Allemands ${ }^{73}$, le taux descend à $13 \%$, moyenne nationale observée par François Lebrun ${ }^{74}$.

20 Il faut maintenant évoquer "l'affaire d'Hirsingue " ${ }^{75}$. Le lundi de Pentecôte, 9 juin 1794, le curé constitutionnel d'Hirsingue célèbre, comme de coutume, la fête locale de Saint Fortuné. La fête religieuse se poursuit par des danses et de libations. Mais quelques individus arrachent l'arbre de liberté et coupent la cime qu'ils jettent devant la maison de l'agent national. Prenant prétexte de cet incident local et en jouant sur l'amalgame entre prêtres et agioteurs, l'administration révolutionnaire décide 
l'arrestation de 285 ministres de culte, sans aucune distinction. Les districts de Belfort et d'Altkirch, pourtant antisémites, ne s'en prennent qu'aux prêtres catholiques. Dans le district de Colmar, l'administrateur du département, le luthérien Metzger ${ }^{76}$, obtient la liberté des pasteurs protestants. Dans le district de Strasbourg, on arrête indistinctement les ministres catholiques, protestants et juifs; dans le district de Benfeld, les ministres catholiques et protestants, mais aucun rabbin. La prison de Belfort sert de lieu de regroupement pour 227 individus.

Les conditions de détention sont dures : vingt détenus par chambre, d'autres dans un dortoir de fortune installé dans l'église de la Citadelle de Besançon, un canon pointé sur les prisonniers, l'absence de latrines, remplacées dans chaque pièce par un baquet. Les gardes tentent d'extorquer des abdications aux prisonniers contre promesse de liberté. Les 37 abdications relevées de juillet à novembre 1794, dont 26 pour le seul mois de juillet, s'expliquent vraisemblablement par le souci d'échapper à la réclusion ou d'en abréger la durée. L'affaire d'Hirsingue a pour conséquence l'amalgame du clergé constitutionnel au clergé réfractaire aux yeux des autorités.

Reste le prix du sang. Six prêtres sont tués ou exécutés, trois dans le Haut-Rhin, trois dans le Bas-Rhin. Dans le Haut-Rhin, Pescheur se déporte ; surpris par une sentinelle française à la frontière, il est abattu le 24 septembre 1792. Joseph Thomas est traduit devant le tribunal pour émigration, condamné à mort et exécuté le 11 décembre 1793. Bernard, déporté, rentré avec un faux passeport, est arrêté et exécuté à Colmar le 11 novembre 1794. Dans le Bas-Rhin, deux ecclésiastiques sont arrêtés lors du départ précipité des Autrichiens occupant Haguenau ${ }^{77}$. Conduits à Strasbourg, Beck est exécuté le 24 décembre 1793 et Frey le 1er janvier 1794. Un troisième, Wolfert, arrêté sous un déguisement féminin, alors qu'il rendait visite à un malade, est guillotiné le 2 juin 1794 avec les deux blanchisseuses qui l'hébergeaient. Si nous sommes loin du massacre des prêtres, comme à Paris, essentiellement parce que le franchissement de la frontière proche permet de se sauver ${ }^{78}$, il n'en reste pas moins que ces ecclésiastiques deviennent des "martyrs de la foi ", dont le souvenir est perpétué ${ }^{79}$. Aux six prêtres, il faut ajouter dix-neuf laïcs exécutés en 1793 et 1794 pour des motifs religieux : cinq dans le Haut-Rhin, quatorze dans le Bas-Rhin, dont deux femmes. Parmi eux, le maire et l'instituteur de Wolschwiller parce qu'ils avaient assisté à la messe dans le grenier de la cure, le 3 décembre 1793 ou le maire de Pfaffenheim ${ }^{80}$ qui avait caché un prêtre réfractaire, exécuté le 2 janvier 1794 ou encore un tisserand parti en pèlerinage à Mariastein en Suisse, guillotiné à son retour le 28 juin 1794.

Le 20 avril 1794, un représentant en mission dans le département du Haut-Rhin, relate qu'il vient de parcourir le département du Haut-Rhin, où il s'était rendu quelques mois auparavant : "J'étais à cette époque assez satisfait des progrès que l'esprit public $\mathrm{y}$ avait faits, surtout quand je le comparais à celui du Bas-Rhin, que je trouvais plus autrichien que républicain. Mais les choses y ont grandement changé de face. [...] Les prêtres, qui y sont nombreux, [...] y deviennent plus dangereux que jamais, fanatisent ouvertement et avec audace. Les églises, au lieu de les convertir en des temples de la Raison, sont parfaitement décorées de tous les signes de la superstition. Les messes s'y disent chaque jour, et le peuple s'y assemble le soir au son de la cloche pour des prières publiques. Les dimanches sont strictement observés, ce qui enlève à la culture et aux transports un temps précieux, irréparable, et porte au commerce un coup sensible, parce que les manufactures chôment ${ }^{81}$. Pour Roland Marx et Freddy Raphaël, c'est la 
lutte antireligieuse menée par la Révolution qui constitue le principal fondement de l'hostilité que l'Alsace manifeste à l'égard des bouleversements nationaux ${ }^{82}$.

2) Le repli protestant

Selon Bernard Vogler, une rupture apparaît dès la mort de Louis XVI ${ }^{83}$. Les protestants semblent mal accepter la chute de la royauté. Désormais, les jacobins les traitent « d'aristocrates » et de «traitres ». Le journal Argos, dirigé par l'ex-vicaire épiscopal constitutionnel, devenu accusateur public, Euloge Schneider, multiplie les attaques contre les protestants. En avril 1793, un arrêté exige de tous les pasteurs et enseignants un certificat de civisme. À l'automne 1793, les protestants ne sont pas épargnés par la politique antireligieuse de la Convention qui interrompt le culte presque partout. Toutes les églises sont fermées. À Strasbourg, le Temple-Neuf voit ses bancs et chaire enlevés et devient un magasin de fourrage puis une étable à cochons, Saint-Guillaume et Saint-Pierre-le-Jeune des dépôts de paille, Saint-Pierre-le-Vieux un dépôt de viandes salées, Saint-Thomas un magasin militaire, le maréchal de Saxe étant coiffé d'un bonnet phrygien, Saint-Nicolas une étable à vaches et à cochons, puis un magasin d'habillement. Les objets liturgiques sont confisqués. L'exercice du culte disparaît totalement dans le Bas-Rhin, subsiste clandestinement dans le Haut-Rhin.

Les pressions suivent pour obtenir des pasteurs l'abjuration. Ceux qui refusent sont emprisonnés au grand séminaire de Strasbourg, comme Blessig ou Haffner, où ils côtoient des curés. Le pasteur Jean Jacques Fischer de Dorlisheim est condamné à mort pour une parole imprudente (" D'où vient ce qu'on entend plus crier Vive la Nation », après l'entrée des alliés en Alsace) et est guillotiné le jour même le 24 novembre 1793. Jean-Michel Lobstein meurt en prison. Certains pasteurs émigrent, d'autres changent de profession: David Cunier devient député, sous-préfet, tandis que Dentzel embrasse une carrière militaire jusqu'au grade de général. Selon Rodolphe Reuss, un pasteur sur dix (22 sur 220) quitte son état ${ }^{84}$. Philippe Juncker, pasteur d'obenheim, exprime sa joie de se « défaire d'un état qui n'avait jamais été de son goût » et « abjure de bonne foi et d'un front serein ce service atroce ». Mathias Engel déclare que le ministère auquel il renonce volontiers ne lui laisserait aucun regret ${ }^{85}$.

Quelques pasteurs camouflent les cultes en séances patriotiques. Schaller de Pfaffenhoffen compose pour la société populaire d'Ingwiller un recueil de chants moraux qui sont quasiment des cantiques. Oberlin fonde un club où il prêche la croisade contre les tyrans qui menacent d'asservir ses ouailles. En juillet 1794, un arrêté des représentants en mission impose l'arrestation de tous les ministres du culte, après l'affaire d'Hirsingue. Dans la pratique, seule une dizaine de pasteurs est écrouée à Besançon. Oberlin bénéficie de nombreuses attestations de civisme et est relâché à Sélestat. De manière générale, les fidèles protestants paraissent moins atteints que les catholiques, puisqu'ils continuent à pratiquer en famille à la maison. Même si les protestants restent en majorité à l'écart de la célébration du culte de la Raison, puis de l'Être Suprême, des exceptions doivent être mentionnées. Le docteur Benjamin Gloxin préside la Société populaire des Amis de la Liberté et de l'Égalité et participe au culte de la Raison, pour lequel Pfeffel ${ }^{86}$ a composé un hymne qui exalte la Raison, fille de Dieu. Le pasteur Lucé magnifie les vertus civiques. Profitant des embarras des catholiques, les protestants cherchent à accroître leurs avantages dans les églises mixtes ${ }^{87}$ où, il est vrai, ils étaient sur le recul pendant tout le XVIIIe siècle.

Pour finir, cette déclaration : à la Société de Strasbourg, le 25 décembre 1793, un membre déclame que «Jésus Christ était le plus grand charlatan qui ait jamais existé. 
Les officiers municipaux Jung et Butenschœn [luthériens d'origine] prennent la défense non de Jésus catholique, apostolique et romain, mais de Jésus-Christ sans culotte prêchant les principes sacrés de l'égalité et une morale sévère" ${ }^{88}$. Comment interpréter ce patriotisme?

3) Une nouvelle vague d'antisémitisme

Comme dans toute période de crise, l'antisémitisme s'affirme avec une vigueur nouvelle. Au sein même des sociétés populaires, on dénonce désormais les juifs " qui font un peuple à part dans la République » ${ }^{89}$ et on affirme : «Bannissez les juifs et vous aurez cassé bras et jambes à l'agiotage $"{ }^{90}$. Les juifs sont accusés de profiter de "l'émancipation pour mieux asseoir leurs affaires, de refuser d'abandonner leurs superstitions, de ne pas accepter de se fondre dans la masse. Alors que la raison triomphe, ils restent attachés à leurs usages barbares et à leur loi inhumaine d'opérer sanguinairement sur l'enfant mâle qui naît ». À cela s'ajoute qu'ils portent la barbe longue "par ostentation et pour singer les patriarches ", qu'ils pratiquent une langue " qu'ils ne connaissent pas et qui n'est plus usitée depuis longtemps ». Il est demandé à la Commission provisoire «de leur interdire ces usages et d'ordonner qu'un autodafé sera fait [...] de tous les livres hébreux et principalement du Talmuth ${ }^{91}$.

Notons que ces mesures s'insèrent dans un ensemble plus général. Dans le Haut-Rhin, on s'en prend à la fois à la barbe des capucins, des juifs et des anabaptistes. Le 13 janvier 1794, la municipalité de Hagenthal-le-Bas arrête que «les juifs devront couper leurs barbes, ne plus porter en public leurs décalogues, ne plus se ceindre publiquement d'un mouchoir et d'une manière générale supprimer tous les signes extérieurs de leur culte ». Les jacobins d'Altkirch invitent le département «à prendre toutes les mesures qui sont en leur pouvoir pour obliger les juif à satisfaire aux lois, de même que les anabaptistes, qui portent également la barbe par pure dévotion quoi que d'ailleurs leur culte soit infiniment plus rapproché de la saine Philosophie et de la Raison $"{ }^{92}$.

Comme les catholiques et les luthériens, les juifs voient quelques-uns des leurs jetés en prison. En 1793, Samuel Seligmann Alexandre et Abraham Auerbach se plaignent de leur détention à la prison du séminaire de Strasbourg, rappelant qu'ils s'étaient appliqués «à former la jeunesse juive selon l'esprit des décrets aux travaux de l'art, dans leurs fabriques respectives de draps et de tabacs ${ }^{93}$. Et, à la société populaire de Colmar, en 1794, un membre demande: "Les juifs portent-ils le signe de leur culte ? ${ }^{94}$.

III - Les derniers soubresauts (1795-1802)

31 Une fois libérés par petits groupes et autorisés à rentrer chez eux, les ecclésiastiques catholiques, protestants et juifs, après un temps d'expectative, vont reprendre leurs fonctions, d'abord timidement, puis ouvertement. Le raidissement consécutif au coup d'État du 18 fructidor an V, avec l'instauration du serment de " haine à la royauté " entraîne une nouvelle vague de tracasserie.

1) Deux clergés catholiques face à face

32 À Banvillars, l'examen du registre de catholicité prouve que le ministère reprend le 13 août 1794 , immédiatement après que le curé est libéré de la citadelle de Besançon ${ }^{95}$. Les nouveaux-nés sont tout de suite baptisés. La reprise du culte au grand jour n'est réelle que fin avril 1795. À Colmar, les premières messes sont célébrées dans des maisons privées à partir du 25 mars 1795, les premiers cultes luthériens à partir du 12 avril 1795, la première messe du curé constitutionnel Graff dans une église le 14 juin 1795. Deux 
serments successifs de "soumission aux lois " sont exigés. 67 prêtres catholiques le prêtent en juin 1795, 33 en juillet 1795, 10 en août 1795. La moitié des actes est effectuée dans la zone belfortaine favorable à la Révolution. Le coup d'État de fructidor an V entraîne l'exigence d'un nouveau serment : 241 ecclésiastiques le prêtent, dont 221 au moins avaient jadis prêté le serment de Liberté-Égalité. Le serment est aussi exigé des ex-religieuses.

Si une poignée de réfractaires finit par le prêter, les gros bataillons de prêtres approuvés et rentrés au printemps 1797 repassent le Rhin dans des proportions qu'il est illusoire de vouloir mesurer. Ils se conforment par là à l'attitude prônée par une partie des évêques émigrés et par les instructions qui circulent de main en main. Quelques réfractaires (mais dans quelle proportion?) préfèrent rester et œuvrer dans la clandestinité ou ne savent pas où aller outre-Rhin. En fait bien des Alsaciens restent proches de la frontière. En février 1794, sur 109 prêtres réfugiés à Einsiedeln en Suisse, 47 sont des Alsaciens ${ }^{96}$. Des allées et venues considérables sont notées aux postes frontières. Parallèlement se met en place la mission du Bas-Rhin et celle du Haut-Rhin organisées par des commissaires épiscopaux ${ }^{97}$. Celle du Haut-Rhin, organisée par Didner s'appuyant sur treize missionnaires en chef, se calque sur l'organisation de Linsolas pour le diocèse de Lyon ${ }^{98}$. L'action des missionnaires contribue à attiser la haine et les divergences du clergé, y compris du clergé réfractaire resté sur place et devant s'accommoder des situations, peu enclin aux leçons de prêtres à l'abri derrière la frontière.

Alors que le clergé réfractaire s'organise pour la reconquête, les constitutionnels relèvent aussi la tête et se donnent un évêque dynamique et entreprenant. Le 24 avril 1796, Marc Antoine Berdolet ${ }^{99}$ devient évêque constitutionnel du Haut-Rhin. Ne voulant point partager la collégiale de Colmar avec les réfractaires et refusant l'humiliation de se voir cantonner à l'église des dominicains, Berdolet préfère dans un premier temps demeurer chez lui à Phaffans, avant de s'installer en octobre 1798 à Soultz, bourgade moins excentrée. Son clergé atteint un maximum de 160 prêtres. Pour faire appliquer les décisions du premier concile national, Berdolet convoque à Soultz pour le 16 mai 1798 un premier synode diocésain. Le rapide voyage que Grégoire fait en octobre 1799 dans le Haut-Rhin dynamise le clergé constitutionnel, lequel se réunit en un deuxième synode du 26 au 29 mai 1800.

Si le clergé constitutionnel du Haut-Rhin relève la tête et se réorganise, il n'en est pas de même dans les deux départements voisins du Bas-Rhin et du Mont-Terrible. Le devenir de ces deux «églises veuves » est bientôt confié à Berdolet, après la démission de l'insipide Brendel le 26 juin 1797. Si le clergé constitutionnel ne paraît pas organisé, il est pourtant bien présent (une cinquantaine de prêtres disséminés dans le département), alors même qu'aucune élection épiscopale n'y est organisée, cas unique en France. Malgré ses efforts, Berdolet ne réussit pas dans son œuvre pastorale pour le Bas-Rhin. La matricule de mai 1800 donne la géographie de l'Église constitutionnelle du Haut-Rhin ${ }^{100}$. Parmi les 157 prêtres en fonctions, 57 œuvrent dans l'arrondissement d'Altkirch. 29 individus sur ces 157 avaient abdiqué le sacerdoce sous la Terreur.

Rappelant le contexte de la guerre, la chasse aux réfractaires n'en est pas moins menée. Mais souvent l'administration locale déplore son impuissance. «Je n'ai pas ignoré qu'il y a des prêtres réfractaires dans la commune de Ribeauvillé. J'y ai envoyé jusqu'à un bataillon entier et ce à plusieurs reprises. Aucun prêtre n'a été arrêté», indique le directoire du Haut-Rhin au ministre de la Police le 6 décembre $1796{ }^{101}$. Les ballons 
vosgiens, mais aussi les importants massifs forestiers de la plaine, fournissent des caches. Mais les opérations de police ne se soldent pas toujours par des échecs. Des arrestations envoient 23 prêtres au moins en déportation (18 à l'île de Ré, 3 à Rochefort, 2 en Guyane), quelques autres en détention à Strasbourg et à Colmar, et deux à la mort (Stackler et Bochelen). Quelques prêtres arrêtés sont libérés par leurs fidèles au cours d'opérations commandos.

Relevons pour finir que la tradition historiographique catholique voudrait encore aujourd'hui faire admettre la vénération pour le « bon prêtre » réfractaire et le rejet de l'infâme constitutionnel. Les choses sont moins simples. On a d'abord cherché un prêtre là où il pouvait s'en trouver un. Quand les ruraux accouraient chez les prémontrés assermentés de Saint-Nicolas à Haguenau ${ }^{102}$, savaient-ils s'ils avaient affaire à un réfractaire ou à un constitutionnel ? Une question qui souligne que le grand absent de l'historiographie alsacienne est non le prêtre, mais bien le fidèle. Les états des lieux et des bilans plus conséquents sur les «indicateurs » des mutations et les pratiques des croyants dans la ligne du colloque de Chantilly (1986) font encore cruellement défaut.

2) La volonté protestante de reconstruire

Après la chute de Robespierre, les suspects ecclésiastiques qui avaient été incarcérés sont relâchés à l'automne 1794. Mis à part Koch qui siège comme administrateur du Bas-Rhin, les autres notables protestants se retirent des affaires publiques. Parmi les députés bas-rhinois sous le Directoire, 6 sur 21 sont d'origine protestante; dans le Haut-Rhin, seul un (Jean Ulrich Metzger) l'est. En 1799, Laurent et André proposent au Conseil des Cinq-Cents la confiscation des biens de la Confession d'Ausbourg et de la Confession Helvétique. La résolution votée par la majorité de la Chambre n'est pas examinée par le Conseil des Anciens en raison du coup d'État du 18 brumaire perpétré par Napoléon Bonaparte. Peut-on admettre que les protestants, considérés en 1796 par le ministre de la Police comme «les seuls patriotes dans ce département» (du BasRhin), sont les vrais vainqueurs de la Révolution en Alsace?

La reprise du culte s'accompagne d'une œuvre de reconstrution administrative entreprise par Blessig, aidé de Haffner, Hebeisen, Frœreisen ${ }^{103}$. La plupart des anciennes paroisses sont reconstituées par l'élection d'un nouveau conseil presbytéral. Se met en place un organisme central où siègent des délégués des sept paroisses strasbourgeoises et qui fonctionne de 1795 à 1801. Selon Jean Frédéric Aufschlager, témoin de l'époque, «bien que les églises furent réouvertes au bout de dix huit mois, les pasteurs étaient en grande partie déconsidérés, l'Église protestante désorganisée. Il n'y avait plus d'autorité, la croyance du peuple était ébranlée » 104.

Bernard Vogler remarque que le corps pastoral perd l'essentiel de ses revenus et que de nombreuses paroisses paient chichement leur pasteur ${ }^{105}$. "Les communes de la campagne choisissaient les pasteurs à leur gré, les payaient mesquinement, leur causaient toutes sortes d'embarras et de déboires, au point que plusieurs d'entre eux abandonnèrent leur fonction ». Selon le professeur allemand Meiners qui revient en Alsace en 1801, après y avoir séjourné en 1789, aucun pasteur n'a plus de 600 florins de revenus. «Dès qu'un étudiant en rupture d'académie vient offrir aux paysans d'exercer les fonctions pastorales pour une somme inférieure à celle qu'ils déboursaient jusqu'ici on congédie le ministre en fonctions, comme on paie ses gages à un domestique renvoyé " ${ }^{106}$. Cette misère matérielle et morale entraîne un effondrement des vocations, d'autant qu'il n'existe plus de formation pour les jeunes pasteurs de 1792 à 1803. Notons que Reinhard Spach, pasteur à Obermodern depuis 1782, se voit évincé par 
l'assemblée des paroissiens qui lui reprochent son «comportement antirépublicain ». Devant son refus de quitter sa paroisse, quelques hommes jettent toutes ses affaires par les fenêtres du presbytère ${ }^{107}$.

41 Un cas particulier est celui des calvinistes de Mulhouse, ville alliée des cantons helvétiques, lesquels ne restent pas insensibles aux idéaux révolutionnaires. De nombreux Mulhousiens se sont affiliés à des sociétés révolutionnaires, en particulier à celles de Colmar et de Thann, où figurent quatre Dollfuss. Selon Marguerite Spoerlin ${ }^{108}$, « la jeune génération désirait, voulait et travaillait de toutes ses forces à la réunion de la France »; c'est l'opportuniste luthérien Metzger qui reçoit les clés de la ville, qui « se donne à la France ». Subtile langue de bois ?

3) Un avenir meilleur pour les juifs?

42 La chute de Robespierre, le 27 juillet 1794, ne calme pas l'antipathie des chrétiens contre les israélites provoquée par la venue croissante des juifs s'établissant dans les villes (avant 1789, ils étaient nombreux à Wintzenheim aux portes de Colmar, où ils étaient interdits de séjour). La confiscation des biens nationaux, la vente quotidienne des hardes et des meubles d'émigrés, les trafics qui permettent le cours variable de la monnaie fiduciaire et la rareté des espèces attirent les juifs dans les grandes villes, surtout Strasbourg et Colmar. On reproche maintenant aux juifs de jouer un rôle trop actif dans la vente des biens nationaux ${ }^{109}$. Roland Marx montre en fait qu'ils sont plutôt des intermédiaires, des acquéreurs et revendeurs de seconde ou de troisième main, d'où une fausse impression d'accaparement par les juifs de biens d'Église - démenti par les faits - qui nourrit l'antisémitisme ${ }^{110}$.

43 Si la présence continuelle des armées, soit à proximité des frontières, soit en Alsace, permet aux plus habiles des juifs de passer des marchés et de faire des bénéfices, une des conséquences de la Révolution reste la ruine de nombreuses familles juives. La multiplication des dépôts de bilan, le contenu des inventaires après décès témoignent de l'appauvrissement de familles entières, ayant misé sur l'assignat ${ }^{111}$. Globalement, François Delpech plaide plutôt l'intégration que le rejet des juifs ${ }^{112}$.

En conclusion, comme le souligne Georges Livet, l'influence de la guerre étrangère va être déterminante sur l'évolution de la Révolution en Alsace ${ }^{113}$. La guerre ouverte fausse les perspectives, commande les comportements, dicte les attitudes excessives, la peur et la violence, tandis que la rumeur court. Le Strasbourg de la Terreur apprend la mort du maire luthérien Dietrich (29 décembre 1793), puis celle de son accusateur, le prêtre catholique abdicataire Euloge Schneider (1er avril 1794), tout un symbole. Les courants (franc-maçonnerie, illuminisme politique et philosophique, jacobinismes français et allemands) s'entrechoquent plus qu'ils ne se croisent. La " Grande Fuite », en décembre 1793, au moment du repli des armées autrichiennes, entraîne avec elle des populations craignant les représailles. Se posent constamment, pendant cette période, les problèmes de départ et de retour. L'expansion révolutionnaire ouvre aux Alsaciens possédant les deux langues les postes de responsabilités dans l'administration et dans l'armée (Kellermann, Lefebvre, Kléber), où l'attrait du panache et de la promotion sociale l'emporte sur le rôle politique. Et la frontière joue un rôle d'une pompe aspirante et refoulante.

Les manifestations paroxystiques de la période révolutionnaire constituent-elles l'écume d'une vague, voire une lame de fond, du phénomène plus général de la déchristianisation étudié depuis une trentaine d'années ${ }^{114}$ ? Au-delà des événements rapportés ci-dessus, il faut souligner que l'Alsace a toujours constitué un pôle de 
résistance à ce phénomène ${ }^{115}$ - à cause du métissage religieux observé - au moins jusqu'en 1975, date depuis laquelle on assiste à une érosion régulière (et irréversible ?) de la pratique religieuse.

Reste enfin à évoquer un double paradoxe historiographique. Le premier concerne l'historiographie régionale. La pléthore d'études sur le thème « religion et Révolution en Alsace ", citées à satiété en notes, évoque une histoire des communautés vue sous l'angle de la persécution, malgré des nuances, et non sous l'angle de la participation à la "régénération» politique et morale. Les comportements des constitutionnels, la mobilisation autour des cultes patriotiques ${ }^{116}$, les tentatives de syncrétisme entre Évangile et Révolution y sont largement minorés. Dès lors, cette page d'histoire n'estelle pas à réécrire? Le second paradoxe relève de l'historiographie nationale. En dépit de la surabondance des travaux, l'Alsace apparaît en définitive peu dans les grandes synthèses ${ }^{117}$, si ce n'est que pour préciser son caractère un peu exceptionnel. Le modèle alsacien serait-il en contradiction avec le modèle français ?

\section{NOTES}

1.Monique CUBELLS, La Révolution française. La guerre et la frontière, Paris, Comité des travaux historiques et scientifiques, 1998.

2.Claude LANGloIs, Michel Vovelle, Timothy TACKETt, Atlas historique de la Révolution Française, t. 9, Religion, Paris, 1996.

3.Monique cuBELLS, La Révolution française. La guerre et la frontière, Paris, Comité des travaux historiques et scientifiques, 1998.

4.Claude LANGloIs, Michel Vovelle, Timothy TACKETt, Atlas historique de la Révolution Française, t. 9, Religion, Paris, 1996.

5.Lise Pommois, La guerre de 1701 à 1714 dans le Ried Nord, Drusenheim, 2001, 352 p.

6.Jean-Michel BOEHLER, Une société rurale en pays rhénan : la paysannerie de la plaine d'Alsace (1648 - 1789), Strasbourg, 1994, $2469 \mathrm{p}$.

7.Bernard VOGLER et Michel HAU, Histoire économique de l'Alsace, Strasbourg, 1997, 430 p.

8. René EPP, Marc LIENHARD et Freddy RAPHAËL, Catholiques, protestants, juifs en Alsace, Colmar, 1992, 238 p. ; Bernard VOGLER, Histoire des chrétiens d'Alsace, Tournai, 1994, 429 p. 9.Jacques GodECHOT, Les Révolutions (1770-1799), Paris, P.U.F., 1963 ; Michel VovelLE, La Révolution française : images et récits (1789-1799), Paris, 1986, 5 volumes.

10. Bernard PLONGERON, Conscience religieuse en Révolution, Paris, 1969 ; Dominique VARRY et Claude MULLER, Hommes de Dieu et Révolution en Alsace, Paris, Éditions Brepols, 1993, $326 \mathrm{p}$.

11.Louis CHÂTELLIER, Tradition chrétienne et renouveau catholique dans l'ancien diocèse de Strasbourg (1650-1770), Paris, 1981, 530 p., id., Religion et piété en Alsace et Lorraine (XVIIe XVIIIe siècles), $\mathrm{n}^{\circ}$ spécial Annales de l'Est, 2003, 303 p.

12.Louis KAMMERER, Répertoire du clergé d'Alsace sous l'Ancien Régime (1648-1792), Strasbourg, 1983, 397 p. (dactylographié).

13.Claude MULLER, Les ordres mendiants en Alsace au XVIIIe siècle, Haguenau, 1984, 350 p. 
14.André SCHAER, Le clergé paroissial catholique en Haute-Alsace sous l'Ancien Régime (1648-1789), Paris, 1966, $294 \mathrm{p}$.

15.Louis CHÂTELLIER, « Rohan », Nouveau Dictionnaire de Biographie Alsacienne, t. 32, Strasbourg, 1998, pp. 3265-3271.

16. Claude Muller, « Mgr Simon Nicolas de Montjoie, les Klinglin et les Gobel », Revue d'Alsace, t. 128, 2002, pp. 281-313.

17.Roland MARX, Recherches sur la vie politique de l'Alsace prérévolutionnaire et révolutionnaire, Strasbourg, 1966, 199 p.

18.François BURCKARD, Le Conseil souverain d'Alsace au XVIIIe siècle, Strasbourg, 1995, 462 p.

19. Claude MulLER et Jean-Luc EiCHENLAuB, Messieurs. Les magistrats du Conseil souverain d'Alsace et leurs familles au XVIIIe siècle, Riquewihr 1998, 270 p. : à 149 magistrats correspondent au moins 321 ecclésiastiques.

20.Claude MULLER, " Aube et robe en Sundgau », Annuaire de la société d'histoire du Sundgau, 1998, pp. 65 - 78 ; Claude MULLER et Marie-Thérèse RILLIOT, « Aube et robe en pays belfortain au XVIIIe siècles ", Bulletin de la société belfortaine d'émulation, n89, 1998, pp. 83-94.

21.Jean-Georges GUTH, Les protestants de Strasbourg sous la monarchie française (1681-1789): une communauté religieuse distincte ?, Strasbourg, 1997, 983 p. (dactylographié) ; Henri STROHL, Le protestantisme en Alsace, Strasbourg 1950, 508 p. ; Jurgen VOSS, Jean-Daniel Schoepflin (1694-1771). Un Alsacien de l'Europe des Lumières, Strasbourg, 1989, 386 p.

22.Thiébaut WALTER, «L'abbé Vogelgsang de Rouffach (1761 - 1844) », Revue d'Alsace, 1929, pp. 120-127, 219-230, 365-375, 805-824.

23.Jean STAROBINSKI, L'invention de la liberté, Genève, 1964, p. 116.

24.Fernand L'HUILLIER, « L'Église d'Alsace pendant la Révolution », Saisons d'Alsace, n9, 1964, p. 44.

25. Rodolphe REUSS, La constitution civile du clergé et la crise religieuse en Alsace, Strasbourg, 1922,378 et 344 p. « Il y a beaucoup de fermentation à Strasbourg par rapport aux lois de la Constitution Civile du Clergé », cf. Revue catholique d'Alsace, 1886, p. 118.

26.Jörg SIEGER, Kardinal im Schatten der Revolution, Kehl, 1986, 414 p.

27.Romain FEIST, « Evangelische und Katholische Kirchenmusik in Strassburg in den zwei letzten Jahrzehnten vor der französische Revolution », Mitteilungen der Arbeitesgemeinschaft für mittelrheinische Musikgeschichte, t. 58, 1992, pp. 330-341.

28. René EPP, « Le Bas-Rhin, département français au pourcentage de prêtres jureurs le plus faible ", Revue d'Alsace, t. 116, 1989-1990, pp. 237-244.

29.L'Alsace et la Révolution, C.R.D.P., Strasbourg, 1972, t. I, p. 4.

30.Médard BARTH, « Die Wahl Brendels zum Bischof von Straßburg », Bulletin ecclésiastique du diocèse de Strasbourg, t. 45, 1926, pp. 569-572 ; t. 46, 1927, pp. 17-21, 90-94, 115-119, 130-139.

31.Louis KAMMERER, « Les prêtres allemands dans le clergé constitutionnel en Alsace ", Revue d'Alsace, t. 116, 1989-1990, pp. 285-290.

32.Claude BETZINGER, Vie et mort d'Euloge Schneider. Des Lumières à la Terreur (1756-1794), Strasbourg 1997, 400 p., id., « Euloge Schneider face à l'histoire », Revue d'Alsace, t. 124, 1998, pp. 141-186.

33. Claude MULLER, « Arbogast Martin », Nouveau Dictionnaire de Biographie Alsacienne (=N.D.B.A.), n²5, 1995, pp. 2533-2535. 
34.Louis KAMMERER, « Jean Baptiste Gobel », N.D.B.A., n¹3, 1988, pp. 1208-1210.

35.Dominique VARRY, " Le clergé du Haut-Rhin face aux serments révolutionnaires », Revue d'Alsace, t. 116, 1989-1990, pp. 217-236.

36.Jules JoAснIм, «L'élection de l'évêque constitutionnel du Haut-Rhin (1791) », Archives de l'Église d'Alsace, t. 19, 1949-1950, pp. 285-329 ; « Jean Georges Gelin, curé

constitutionnel de Guémar ", Bulletin de la société d'histoire de Ribeauvillé, 1950, pp. 32-47 ; «La crise religieuse à Belfort pendant la Révolution (1789-1802) », Bulletin de la société belfortaise d'émulation, 1956-1957 ; « Le séminaire constitutionnel de Colmar (1791-1792) », Annuaire de la société d'histoire de Colmar, 1953, pp. 124-139 ; « L'installation à Colmar de l'évéché constitutionnel du Haut-Rhin (1791-1793) », Annuaire de la société d'histoire de Colmar, 1954, pp. 112-127.

37.

38. Claude MULLER, Les cisterciens d'Alsace dans la tourmente révolutionnaire, t. 23 de la Documentation Cistercienne, Wetteren, 1988, 137 p. (85 notices biographiques) ; Les augustins d'Alsace dans la tourmente révolutionnaire, Éditions Guéniot, Langres, 1991, 131 p. (98 notices) ; Les bénédictins d'Alsace dans la tourmente révolutionnaire, Langres, 1991, 187 p. (106 notices) ; Les dominicains d'Alsace dans la tourmente révolutionnaire, Langres, 1991, 113 p. (69 notices) ; « Les récollets d'Alsace dans la tourmente révolutionnaire ", Archivum Franciscanum Historicum, t. 83, 1990, pp. 421-461 ; t. 84, 1991, pp. 365-405 ; t. 94, 2001, pp. 401-435 ; t. 95, 2002, pp. 399-432 (241 notices) ; « Une grande mutation des ordres religieux alsaciens (1721-1820)», Religieux et Religieuses pendant la Révolution (1770-1820), Actes du colloque de Lyon (15-17 septembre 1992), Lyon 1995, t. I, pp. 269-286.

39. Claude MULLER, « Religieuses sundgoviennes pendant la Révolution », Annuaire de la société d'histoire du Sundgau, 1999, pp. 309-322 ; « Religieuses originaires d'Obernai et environs pendant la Révolution ", Annuaire de la société d'histoire de Dambach-la-Ville, Barr, Obernai, t. 35, 2001, pp. 141-144 ; «Le destin de neuf religieuses originaires de la région de Thann-Guebwiller pendant la Révolution, Annuaire de la société d'histoire des régions de Thann-Guebwiller, t. 20, 2000-2003, pp. 112-118.

40.A. M. Sélestat, ms 415.

41.Les statistiques des A. N., D XIX, 22 ont le mérite d'exister et sont irremplaçables. Peut-on affirmer que leur fiabilité est sujette à caution? Gonflement voulu des statistiques? Minoration des historiens?

42. Timothy TACKETT, La Révolution, l'Église, la France, le serment de 1791, Paris, 1986, p. 409. 43.Bernard Cousin et alii, Hommes de Dieu et Révolution en Provence, Éditions Brepols, Paris, 1997.

44. Claude MULLER, «Wir haben den selben Herrgott. Luthériens et catholiques en Alsace au XVIIIe siècle ", dans Terres d'Alsace, chemins d'Europe. Mélanges offerts à Bernard Vogler, Presses Universitaires de Strasbourg, 2003, pp. 423-445.

45. Claude MulleR, Colmar au XVIIIe siècle, Strasbourg, 2000, 176 p.

46.Hermann GERST, Histoire de Hunspach et de la paroisse Hunspach-Hoffen. Ingolsheim, Strasbourg, 1975, $256 \mathrm{p}$.

47.Yves TYL, Les cahiers de doléances de Haute-Alsace, Colmar, 1975, t. II, pp. 56, 67, 79-81 ; Robert STEEGMANN, « Les cahiers de doléances en Alsace », Revue d'Alsace, t. 116, 1989-1990, pp. 23-34. 
48.À Strasbourg, Colmar, Munster, Wissembourg et Landau, le magistrat est composé pour moitié de luthériens, pour moitié de catholiques, autre exemple de particularisme alsacien.

49.Archives du Ministère des Affaires Étrangères (A.M.A.E.), Paris, Série Mémoires et Documents, fonds Alsace, t. 58, f 114.

50.Ibid., f 205. Jurgen Voss, « Christophe Guillaume Koch », N.D.B.A., n²1, 1993, pp. 2036-2038.

51.Rodolphe Reuss, Les Églises protestantes d'Alsace pendant la Révolution (1789-1802), Paris, $1906,320 \mathrm{p}$.

52.Bernard VogleR, «Les Protestants d'Alsace et la Révolution », Revue d'Alsace, t. 116, 1989-1990, pp. 197-206. Voir aussi le texte du luthérien de Turckheim, du 9 avril 1791, publié anonymement sous le titre de « Une voix protestante en faveur de la tolérance dans la persécution de 1790 ", Revue Catholique d'Alsace, 1883, pp. 606-608.

53.Hélène GEORGER-VOGT et Jean-Pierre KINTZ, « Frédéric de Dietrich », N.D.B.A., nº 8 , 1986, pp. 653-654.

54.Marcel TномаNN, « Jean Laurent Blessig », N.D.B.A., n4, 1984, pp. 249-252.

55.Frédéric Charles HeITZ, Les sociétés politiques de Strasbourg pendant les années 1790 à 1795, Strasbourg 1863, p. 125.

56.Sylvie GUETH, La vente des biens nationaux dominicains de Colmar (1790-1794), Strasbourg 1994, $102 \mathrm{p}$.

57.Léonard FisCHER, « Fragment des souvenirs d'un Alsacien soldat dans l'armée de Condé (1772-1795) », Revue Catholique d'Alsace, 1893, pp. 256-257.

58.Jean DALTROFF, « La vie économique des juifs d'Alsace à l'époque révolutionnaire », Revue d'Alsace, t. 116, 1989-1990, pp. 173-186.

59.Robert WEYL et Jean DALTROFF, « Le cahier des doléances des juifs d'Alsace », Revue d'Alsace, t. 109, 1983, pp. 65-80.

60.L'Alsace et la Révolution, CRDP Strasbourg, 1972, t. I, p. 85.

61.Bernard Plongeron, L'abbé Grégoire (1750-1831) ou l'arche de la fraternité, Paris, 1989.

62.Freddy RAPHAËL et Robert WeYL, « Medelsheim Cerf Beer », N.D.B.A., n6, 1985, p. 478.

63.René Moulinas, Les juifs du pape en les communautés d'Avignon et du Comtat Venaissin aux 17e et 18e siècles, Toulouse, 1981, $584 \mathrm{p}$.

64. Jean DaltrofF, «Les juifs : un bilan », Revue d'Alsace, 2000, pp. 239-265.

65.L'Alsace et la Révolution, CRDP Strasbourg, 1972, t. I, p. 64. Sur Reubell, N.D.B.A., pp. 3168-3172.

66.Lise Pommois, 1796-1797. Les deux passages du Rhin par l'armée de Rhin-et-Moselle et le siège de Kehl, Drusenheim, 1997, 423 p.

67.Michel VovelLe, La Révolution contre l'Église. De la Raison à l'Être Suprême, Paris, 1988, et Religion et révolution : la déchristianisation de l'An II, Paris, Hachette, 1976, 316 p. Voir aussi le débat historiographique entre Gérard Cholvy et Michel Vovelle dans Annales historiques de la Révolution française, $\mathrm{n}^{\circ} 231,1978$, pp. 451-470 et Serge BIANCHI, « La déchristianisation de l'an II, Essai d'interprétation », id., pp. 341-371.

68.O.R. LANDSMANN (pseudonyme d'Octave RABAÏOYE), « Wissembourg pendant la Révolution ", Revue Catholique d'Alsace, 1895, pp. 334-347.

69.Jules JOACHIM, « La crise religieuse à Belfort pendant la Révolution (1789-1802) », Bulletin de la société belfortaine d'émulation, 1956-1957, pp. 83-114.

70.Claude MULLER, «La paroisse Saint-Georges Haguenau dans la tourmente révolutionnaire ", Études Haguenoviennes, t. 9, 1983, pp. 109-117. 
71.Louis KAMMERER, «Le clergé constitutionnel en Alsace (1791-1803), Archives de l'Église d'Alsace, t. 48, 1989, pp. 1-55, surtout p. 32.

72.Dominique VARRY et Claude MULler, Hommes de Dieu, op. cit., p. 173.

73.Michel Vovelle avait relevé, dans l'historiographie, cette propension à qualifier les prêtres abdicataires d'intrus, d'étrangers, venus d'ailleurs, d'autres diocèses ; cf. Annales Historiques de la Révolution française, 1978, p. 468.

74.François LeBRUN, Histoire des catholiques en France, Paris, 1980, p. 261. Chiffres inférieurs à ceux observés à Paris (Bernard Plongeron), en Provence (Michel Vovelle) et dans le Mont-Terrible (Jean-René Suratteau). Aucune étude sur les « curés rouges » en Alsace, voir Albert SовоuL, "Sur les « curés rouges » dans la Révolution Française », Annales Historiques de la Révolution française, n²49, 1982, pp. 349-363 ; Serge BIANCHI, « Les curés rouges dans la Révolution Française », id., pp. 364-392 et Claude LANGLoIS, "Les vaincus de la Révolution. Jalons pour une sociologie des prêtres mariés », Colloque Albert Mathiez- Georges Lefebvre, Voies nouvelles pour l'histoire de la Révolution Française, Paris, 1978, pp. 282-312, quoique faisable en reprenant les travaux de Louis Kammerer. 75.Jules JOACHIM, « L'affaire d'Hirsingue et l'arrestation des prêtres en 1794 », Archives de l'Église d'Alsace, n²5, 1958, pp. 191-209, n²6, 1959, pp. 211-298; n³3, 1969, pp. 137-158; n³7, 1974, pp. 129-162.

76.Jean-Marie SchмITT, « Jean Ulrich Metzger », N.D.B.A., n 26, 1995, p. 2626.

77.Joseph BRAUNER, Strassburger Priester - Märtyrer der Schreckenzeit (1793 - 1794), Rech, $1922,142 \mathrm{p}$.

78.Isidore Вечснот, «Le clergé de Haute Alsace en exil pendant la Révolution », Revue Catholique d'Alsace, 1895, pp. 533, 599-612, 695-709, 753-765, 823-836, 881-896 ; 1896, pp. 10-17, 88-97, 200-213, 341-349, 494-503, 571-580 ; W. WuHR, Die Emigranten der französischen Revolution in bayerischen und fränkischen Kreis, München, 1938 ; Paulus WEISSENBERGER, « Französische Flüchtingsgeistiche in der Abtei Neresheim/Wurtemberg am Ende des 18. Jahrhunderts (1790-1798) », Archives de l'Église d'Alsace, t. 38, 1975, pp. $345-358$.

79.Quelques monuments, encore visibles de nos jours, cités par Gérard ULRICH et Yves BonNEL, « Les témoins visibles de la Révolution Française en Alsace (1789-1799) », Revue d'Alsace, t. 118, 1989-1990, p. 364.

80.Son procès a été refait en 2002 par la municipalité de Pfaffenheim à grand renfort de médias!

81.L'Alsace et la Révolution, t. I, p.134.

82.Geneviève HERBERICH-MARX et Freddy RAPHAËL, « L'imagerie religieuse durant la Révolution en Alsace ", Revue d'Alsace, t. 116, 1989-1990, p. 340.

83.Bernard VOGLER, «Les protestants et la Révolution », pp. 200-202 et «L'accueil de la Révolution française par les théologiens luthériens de Strasbourg ", Protestantisme et Révolution, 6e colloque Jean Boisset, Montpellier, Sauramps éditions, 1990, pp. 137-146. 84.Roldolphe Reuss, Les Églises protestantes, op. cit., p. 152.

85.Marc LIENHARD, « La foi chrétienne à l'heure de la Révolution : l'itinéraire spirituel et politique du pasteur alsacien Mathias Engel (1755-1811)», Revue d'histoire et de philosophie religieuses, 1989, pp. 451-473.

86.Gabriel BRAEUNER, Pfeffel l'Européen, esprit français et culture allemande en Alsace au siècle des Lumières, Strasbourg, 1994, $222 \mathrm{p}$.

87.Claude MulLER, «Simultaneum », L'Encyclopédie de l'Alsace, t. 11, 1985, pp. 6896-6903. 
88.Frédéric Charles HEITZ, Les sociétés politiques de Strasbourg de 1790 à 1795, Strasbourg, 1863 , p. 316.

89.Roland OBERLÉ, "Strasbourg de la guerre de Trente Ans à Napoléon », dans Georges LIVET et Francis RAPP, Histoire de Strasbourg, Strasbourg, 1981, t. III, p. 586.

90.Paul Leuilliot, Les Jacobins de Colmar, Colmar, 1923, p. 25.

91.Geneviève HERBERICH - MARX - Freddy RAPHAËL, « L'imagerie religieuse », pp. 339-40.

92.Jules JоAснім, « Les signes extérieurs d'un culte (1793-1794) », Annuaire de la Société d'histoire sundgovienne, 1954, pp. 48-54.

93.Jean DALTROFF, « La vie économique des juifs », p. 183.

94.A. D'OCHSSENFELD, «La société populaire révolutionnaire de Colmar en 1794 », Revue Catholique d'Alsace, 1896, p. 423.

95.Dominique VARRY, « La paroisse de Phaffans d'après le registre du curé constitutionnel Berdolet (1789-1805) », dans Bernard PLONGERON, Pratiques religieuses, mentalités et spiritualités dans l'Europe révolutionnaire (1770 - 1820), Turnhout, 1988, pp. 236-245.

96.Isidore Веuснот, « Le clergé de Haute Alsace en exil pendant la Révolution », Revue Catholique d'Alsace, 1895, pp. 761-765.

97.Louis KAMMERER, « Les commissaires épiscopaux dans le diocèse de Strasbourg pendant la Révolution d'après les cahiers de Bert de Majan, curé de Stutzheim et quelques autres documents ", Archives de l'Église d'Alsace, t. 42, 1983, pp. 273-314. 98.Jules Jолснім, « La mission du Haut-Rhin pendant la Révolution », Revue d'Alsace, 1953, pp. 79-88.

99.Georges KNITTEL, « Marc Antoine Berdolet, évêque constitutionnel de Colmar et concordataire d'Aix-la-Chapelle ", Archives de l'Église d'Alsace, t. 37, 1974, pp. 226-244. 100.Joseph LiBLIN, Documents pour servir à l'histoire religieuse en Alsace pendant la Révolution, Mulhouse, 1859, pp. 62-68.

101.Dominique VARRY et Claude MULleR, Hommes de Dieu, op. cit., p. 253.

102.Claude MULLER, «Les prémontrés de Saint-Nicolas Haguenau dans la tourmente révolutionnaire (1789-1802) ", Études Haguenoviennes, t. 15, 1989, pp. 151-162. 103.Bernard VOGLER, «L'accueil de la Révolution Française par les théologiens luthériens de Strasbourg », Protestantisme et Révolution, Montpellier, 1990, pp. 137-146. 104.Jean Frédéric AUFSCHLAGER, Les souvenirs d'un vieux professeur strasbourgeois, Strasbourg, 1893, p. 21.

105.Bernard VOGLER, « Les protestants et la Révolution », art. cit., p. 204.

106. Marcel SCHEIDHAUER, Les Églises luthériennes en France (1800-1815), Strasbourg, 1975, p. 42.

107.Willy GUGGENBUHL, Obermodern. Chronik einer unterelsässischen Landgemeinde, Saverne, 1948, pp. 84-86.

108.Georges LIVET et Raymond OBERLÉ, Histoire de Mulhouse, Strasbourg, 1977, p. 162.

109.Jean Daltroff, « La vie économique des juifs d'Alsace », art. cit., p. 182.

110.Roland MARX, « La Révolution française », Encyclopédie de l'Alsace, t. 10, 1985, p. 6378.

111.Jean DALTROFF, « La vie économique des juifs d'Alsace », art. cit., p. 184.

112.François DeLPECH, «Les juifs en France et dans l'Empire et la genèse du Grand Sanhédrin ", Annales historiques de la Révolution française, n²35, 1979, pp. 1-26. 113.Georges LIVET, «Conclusion », Revue d'Alsace, t. 116, 1989-1990, p. 402. 
114. Gérard CHOLVY et Yves Marie HILAIRE, Histoire religieuse de la France contemporaine, Toulouse, 1986, trois vol., $352+457+549$ p.

115. Claude MULLER, Dieu est catholique et alsacien. La vitalité du diocèse de Strasbourg au XIXe siècle (1802-1914), Strasbourg, 1986, 1209 p. ; Charles MUNIER, René LEVRESSE, René EPP, Histoire de l'Église catholique en Alsace, Strasbourg, 2003, 656 p.

116. Michel Vovelle, La mentalité révolutionnaire, Paris, 1985, 290 p.

117. Claude GEFFRÉ et Jean-Pierre JosSUA (dir.), Französische Revolution und Kirche. Concilium. Internationale Zeitschrift für Theologie, $n^{\circ} 1,1989,93$ p. (contributions de Gérard Cholvy, Pierre Colin, Peter Eicher notamment). Une exception : Die Geschichte des Christentums, t. 10, Aufklärung, Revolution, Restauration (1750-1830), Bernard PLONGERON (dir.), Herder, Fribourg/Brisgau, 2001, 880 p. (qui englobe catholiques, protestants, juifs). Pour terminer, posons les jalons d'une recherche à venir. Quels sont les hommes politiques qui ont ou n'ont pas un ecclésiastique dans leur parenté ? Citons par exemple le plus célèbre Alsacien de la Révolution, magistralement évoqué par Jean-René SURATTEAu et Alain BIschoff, Jean-François Reubell. L'Alsacien de la Révolution française, Éditions du Rhin, 1995, 543 p. Reubell a un oncle et une tante Simottel, respectivement conventuel et dominicaine à Unterlinden de Colmar, jusqu'à présent inconnus de l'historiographie. Bien d'autres révolutionnaires sont dans ce cas. Comment ces hommes politiques se conduisent-ils par rapport à cette parenté « indésirable »? Comment concilient-ils, à leur niveau, religion et révolution?

\section{RÉSUMÉS}

La Révolution s'est-elle déroulée de façon différente en Alsace que dans le reste de la France ? Il serait sans doute prétentieux de l'affirmer, tant le mouvement centralisateur paraît fort à cette époque. Toutefois, deux facteurs y induisent des comportements originaux: d'une part, la présence de la frontière ${ }^{1}$ et en corollaire l'omniprésence de la guerre ; d'autre part, la diversité des religions ${ }^{2}$ pratiquées dans cette région.

Religion and Revolution in Alsace. Did the Revolution unfold differently in Alsace as compared with the rest of France? This might well seem an invidious claim, such was the force of the centralisation movement under way at the time. Nevertheless, two factors were conducive to original behaviour: on the one hand, the nearness of the border ${ }^{3}$ and as a corollary the allpervasive presence of war; and on the other, the diversity of religious practice ${ }^{4}$ prevalent in the region.

\section{INDEX}

Mots-clés : catholicisme, protestantisme, judaïsme, Alsace 